\title{
Pseudo almost automorphic solutions of class $r$ in $\alpha$-norm under the light of measure theory
}

https://doi.org/10.1515/msds-2020-0112

Received June 26, 2020; accepted July 11, 2020

Abstract: Using the spectral decomposition of the phase space developed in Adimy and co-authors, we present a new approach to study weighted pseudo almost automorphic functions in the $\alpha$-norm using the measure theory.

Keywords: Measure theory; ergodicity; $(\mu, v)$-pseudo almost automorphic function; evolution equations; reaction diffusion system; partial functional differential equations.

AMS Subject Classification: 34K14; 34K30; 35B15; 35K57; 44A35; 47D06.

\section{Introduction}

In this work, present a new approach to study weighted pseudo almost automorphic functions in $\alpha$-norm for the following partial functional differential equation

$$
u^{\prime}(t)=-A u(t)+L\left(u_{t}\right)+f(t) \text { for } t \in \mathbb{R},
$$

where $-A: D(A) \rightarrow X$ is the infinitesimal generator of compact analytic semigroup of uniformly bounded linear operators on a Banach space $X, C_{\alpha}=C\left([-r, 0], D\left(A^{\alpha}\right)\right), 0<\alpha<1$, denotes the space of continuous functions from $[-r, 0]$ into $D\left(A^{\alpha}\right), A^{\alpha}$ is the fractional $\alpha$-power of A. This operator $\left(A^{\alpha}, D\left(A^{\alpha}\right)\right)$ will be describe later and

$$
\|\varphi\|_{C_{\alpha}}=\left\|A^{\alpha} \varphi\right\|_{C([-r, 0], X)} .
$$

For $t \geq 0$, and $u \in C\left([-r, a], D\left(A^{\alpha}\right)\right), r>0$ and $u_{t}$ denotes the history function of $C_{\alpha}$ defined by

$$
u_{t}(\theta)=u(t+\theta) \text { for }-r \leq \theta \leq 0 .
$$

$L$ is a bounded linear operator from $C_{\alpha}$ into $X$ and $f: \mathbb{R} \rightarrow X$ is a continuous function.

Some recent contributions concerning pseudo almost periodic solutions for abstract differential equations similar to equation (1) have been made.

In [3], the authors present new approach to study weighted pseudo almost automorphic functions using the measure theory. They present a new concept of weighted ergodic functions which is more general than the classical one. Then they establish many interesting results on the functional space of such functions like completeness and composition theorems. Our aim of this work is to generalize the results obtain in [5].

This work is organised as follow, in section 2 we recall some preliminary results about analytic semigroups and fractional power associated to its generator will be used throughout this work. In section 3, we

\footnotetext{
*Corresponding Author: Issa Zabsonre: Université Joseph KI-ZERBO, Unité de Recherche et de Formation en Sciences Exactes et Appliquées, Département de Mathématiques, B.P.7021 Ouagadougou 03, Burkina Faso, zabsonreissa@yahoo.fr Djendode Mbainadji: Université Joseph KI-ZERBO, Unité de Recherche et de Formation en Sciences Exactes et Appliquées, Département de Mathématiques, B.P.7021 Ouagadougou 03, Burkina Faso, mbainadjidjendode@gmail.com
} 
recall some prelimary results on spectral decomposition. In section 4, we recall some prelimary results on $\alpha-(\mu, v)$-pseudo almost automorphic functions and neutral partial functional differential equations that will be used in this work. In section 5, we give some properties of $\alpha-(\mu, v)$-pseudo almost automorphic functions of class $r$. In section 6 , we discuss the main result of this paper. Using the strict contraction principle we show the existence and uniqueness of $\alpha-(\mu, v)$-pseudo almost automorphic solution of class $r$ for equation (1). Last section is devoted to some applications arising in population dynamics.

\section{Analytic semigroup}

Let $(X,\|\cdot\|)$ be a Banach space and $\alpha$ be a constant such that $0<\alpha<1$ and $-A$ be the infinitesimal generator of a bounded analytic semigroup of linear operator $(T(t))_{t \geq 0}$ on X. We assume without loss of generality that $0 \in \rho(A)$. Note that if the assumption $0 \in \rho(A)$ is not satisfied, one can substitute the operator $\mathrm{A}$ by the operator $(A-\sigma I)$ with $\sigma$ large enough such that $0 \in \rho(A-\sigma I)$. This allows us to define the fractional power $A^{\alpha}$ for $0<\alpha<1$, as a closed linear invertible operator with domain $D\left(A^{\alpha}\right)$ dense in X. The closeness of $A^{\alpha}$ implies that $D\left(A^{\alpha}\right)$, endowed with the graph norm of $A^{\alpha},|x|=\|x\|+\left\|A^{\alpha} x\right\|$, is a Banach space. Since $A^{\alpha}$ is invertible, its graph norm $|$.$| is equivalent to the norm |x|_{\alpha}=\left\|A^{\alpha} x\right\|$. Thus, $D\left(A^{\alpha}\right)$ equipped with the norm |. $\mid \alpha$, is a Banach space, which we denote by $X_{\alpha}$. For $0<\beta \leq \alpha<1$, the imbedding $X_{\alpha} \hookrightarrow X_{\beta}$ is compact if the resolvent operator of A is compact. Also, the following properties are well known.

Proposition 2.1. [8] Let $0<\alpha<1$. Assume that the operator $-A$ is the infinitesimal generator of an analytic semigroup $(T(t))_{t \geq 0}$ on the Banach space $X$ satisfying $0 \in \rho(A)$. Then we have

i) $T(t): X \rightarrow D\left(A^{\alpha}\right)$ for every $t>0$.

ii) $T(t) A^{\alpha} x=A^{\alpha} T(t) x$ for every $x \in D\left(A^{\alpha}\right)$ and $t \geq 0$.

iii) for every $t>0, A^{\alpha} T(t)$ is bounded on $X$ and there exist $M_{\alpha}>0$ and $\omega>0$ such that

$$
\left\|A^{\alpha} T(t)\right\| \leq M_{\alpha} e^{-\omega t} t^{-\alpha} \text { for } t>0 .
$$

iv) If $0<\alpha \leq \beta<1, D\left(A^{\beta}\right) \hookrightarrow D\left(A^{\alpha}\right)$.

v) There exists $N_{\alpha}>0$ such that

$$
\left\|(T(t)-I) A^{-\alpha}\right\| \leq N_{\alpha} t^{\alpha} \text { for } t>0 .
$$

Recall that $A^{-\alpha}$ is given by the following formula

$$
A^{-\alpha}=\frac{1}{\Gamma(\delta)} \int_{0}^{+\infty} t^{\alpha-1} T(t) d t
$$

where the integral converges in the uniform operator topology for every $\alpha>0$.

Consequently, if $T(t)$ is compact for each $t>0$, then $A^{-\alpha}$ is compact.

\section{Spectral decomposition}

To equation (1), we associate the following initial value problem

$$
\left\{\begin{array}{l}
\frac{d}{d t} u(t)=-A u(t)+L\left(u_{t}\right)+f(t) \text { for } t \geq 0 \\
u_{0}=\varphi \in C_{\alpha}
\end{array}\right.
$$

where $f: \mathbb{R}^{+} \rightarrow X$ is a continuous function.

For each $t \geq 0$, we define the linear operator $u(t)$ on $C_{\alpha}$ by

$$
u(t) \varphi=v_{t}(., \varphi)
$$


where $v(., \varphi)$ is the solution of the following homogeneous equation

$$
\left\{\begin{array}{l}
\frac{d}{d t} v(t)=-A v(t)+L\left(v_{t}\right) \text { for } t \geq 0 \\
v_{0}=\varphi \in C_{\alpha}
\end{array}\right.
$$

Proposition 3.1. [1] Let $\mathcal{A}_{u}$ defined on $C_{\alpha}$ by

$$
\left\{\begin{array}{l}
D\left(\mathcal{A}_{u}\right)=\left\{\varphi \in C_{\alpha}, \varphi^{\prime} \in C_{\alpha}, \varphi(0) \in D(A), \varphi(0)^{\prime} \in \overline{D(A)} \text { and } \varphi(0)^{\prime}=-A \varphi(0)+L(\varphi)\right\} \\
\mathcal{A}_{u} \varphi=\varphi^{\prime} \text { for } \varphi \in D\left(\mathcal{A}_{\varkappa}\right) .
\end{array}\right.
$$

Then $\mathcal{A}_{\mathcal{U}}$ is the infinitesimal generator of the semigroup $(U(t))_{t \geq 0}$ on $C_{\alpha}$.

Let $\left\langle X_{0}\right\rangle$ be the space defined by

$$
\left\langle X_{0}\right\rangle=\left\{X_{0} c: c \in X\right\}
$$

where the function $X_{0} c$ is defined by

$$
\left(X_{0} c\right)(\theta)=\left\{\begin{array}{l}
0 \text { if } \theta \in[-r, 0[ \\
c \text { if } \theta=0
\end{array}\right.
$$

Consider the extension $\mathcal{A}_{\mathcal{U}}$ defined on $C_{\alpha} \oplus\left\langle X_{0}\right\rangle$ by

$$
\left\{\begin{array}{l}
D\left(\widetilde{\mathcal{A}_{u}}\right)=\left\{\varphi \in C^{1}\left([-r, 0] ; X_{\alpha}\right): \varphi(0) \in D(A) \text { and } \varphi(0)^{\prime} \in \overline{D(A)}\right\} \\
\widetilde{\mathcal{A}_{u}} \varphi=\varphi^{\prime}+X_{0}\left(A \varphi(0)+L(\varphi)-\varphi(0)^{\prime}\right) .
\end{array}\right.
$$

We make the following assertion:

$\left(\mathbf{H}_{\mathbf{0}}\right)$ The operator $-A$ is the infinitesimal generator of an analytic semigroup $(T(t))_{t \geq 0}$ on the Banach space $\mathrm{X}$ and satisfies $0 \in \rho(A)$.

Lemma 3.2. [2] Assume that $\left(\boldsymbol{H}_{\boldsymbol{O}}\right)$ holds. Then, $\widetilde{\mathcal{A}_{u}}$ satisfies the Hille-Yosida condition on $C_{\alpha} \oplus\left\langle X_{0}\right\rangle$ there exist $\widetilde{M} \geq 0, \widetilde{\omega} \in \mathbb{R}$ such that $] \widetilde{\omega},+\infty\left[\subset \rho\left(\widetilde{\mathcal{A}_{u}}\right)\right.$ and

$$
\left|\left(\lambda I-\widetilde{\mathcal{A}_{u}}\right)^{-n}\right| \leq \frac{\widetilde{M}}{(\lambda-\widetilde{\omega})^{n}} \text { for } n \in \mathbb{N} \text { and } \lambda>\widetilde{\omega} .
$$

Now, we can state the variation of constants formula associated to equation (2).

Theorem 3.3. [1] Assume that $\left(\boldsymbol{H}_{\boldsymbol{O}}\right)$ holds. Then for all $\varphi \in C_{\alpha}$, the solution $u$ of equation (2) is given by the following variation of constants formula

$$
u_{t}=u(t) \varphi+\lim _{\lambda \rightarrow+\infty} \int_{0}^{t} u(t-s) \widetilde{B}_{\lambda}\left(X_{0} f(s)\right) d s \text { for } t \geq 0,
$$

where $\widetilde{B}_{\lambda}=\lambda\left(\lambda I-\widetilde{\mathcal{A}_{U}}\right)^{-1}$.

Definition 3.4. We say that a semigroup $(U(t))_{t \geq 0}$ is hyperbolic if

$$
\sigma\left(\mathcal{A}_{\mathcal{U}}\right) \cap i \mathbb{R}=.
$$


For the sequel, we make the following assumption:

$\left(\mathbf{H}_{\mathbf{1}}\right) T(t)$ is compact on $\overline{D(A)}$ for every $t>0$.

We get the following result on the spectral decomposition of the phase space $C_{\alpha}$.

Proposition 3.5. [1] Assume that $\left(\boldsymbol{H}_{\boldsymbol{O}}\right)$ and $\left(\boldsymbol{H}_{1}\right)$ hold. If the semigroup $(U(t))_{t \geq 0}$ is hyperbolic, then the space $C_{\alpha}$ is decomposed as a direct sum

$$
C_{\alpha}=S \oplus U
$$

of two $\mathcal{U}(t)$ invariant closed subspaces $S$ and $U$ such that the restriction of $(\mathcal{U}(t))_{t \geq 0}$ on $U$ is a group and there exist positive constants $\bar{M}$ and $\omega$ such that

$$
\begin{aligned}
&|\mathcal{U}(t) \varphi|_{C_{\alpha}} \leq \bar{M} e^{-\omega t}|\varphi|_{C_{\alpha}} \text { for } t \geq 0 \text { and } \varphi \in S \\
&|\mathcal{U}(t) \varphi|_{C_{\alpha}} \leq \bar{M} e^{\omega t}|\varphi|_{C_{\alpha}} \text { for } t \leq 0 \text { and } \varphi \in U,
\end{aligned}
$$

where $S$ and $U$ are called respectively the stable and unstable space, $\Pi^{s}$ and $\Pi^{u}$ denote respectively the projection operator on $S$ and $U$.

\section{$4(\mu, v)$-Pseudo almost automorphic functions}

In this section, we recall some properties about pseudo almost automorphic functions. Let $B C(\mathbb{R}, X)$ be the space of all bounded and continuous function from $\mathbb{R}$ to $X$ equipped with the uniform topology norm.

Definition 4.1. A bounded continuous function $\phi: \mathbb{R} \rightarrow X$ is called almost automorphic if for each real sequence $\left(s_{m}\right)$, there exists a subsequence $\left(s_{n}\right)$ such that

$$
g(t)=\lim _{n \rightarrow+\infty} \phi\left(t+s_{n}\right)
$$

is well defined for each $t \in \mathbb{R}$ and

$$
\lim _{n \rightarrow+\infty} g\left(t-s_{n}\right)=\phi(t)
$$

for each $t \in \mathbb{R}$.

We denote by $A A(\mathbb{R}, X)$, the space of all such functions.

Proposition 4.2. [7] $A A(\mathbb{R}, X)$ equipped with the sup norm is a Banach space.

Definition 4.3. Let $X_{1}$ and $X_{2}$ be two Banach spaces. A bounded continuous function $\phi: \mathbb{R} \times X_{1} \rightarrow X_{2}$ is called almost automorphic in $t \in \mathbb{R}$ uniformly for each $x$ in $X_{1}$ if for every real sequence $\left(s_{m}\right)$, there exists a subsequence $\left(s_{n}\right)$ such that

$$
g(t, x)=\lim _{n \rightarrow+\infty} \phi\left(t+s_{n}, x\right) \text { in } X_{2}
$$

is well defined for each $t \in \mathbb{R}$ and each $x \in X_{1}$ and

$$
\lim _{n \rightarrow+\infty} g\left(t-s_{n}, x\right)=\phi(t, x) \text { in } X_{2}
$$

for each $t \in \mathbb{R}$ and for every $x \in X_{1}$.

Denote by $A A\left(\mathbb{R} \times X_{1} ; X_{2}\right)$ the space of all such functions. 
Definition 4.4. A bounded continuous function $\phi: \mathbb{R} \rightarrow X$ is called compact almost automorphic if for each real sequence $\left(s_{m}\right)$, there exists a subsequence $\left(s_{n}\right)$ such that

$$
g(t)=\lim _{n \rightarrow+\infty} \phi\left(t+s_{n}\right) \text { and } \lim _{n \rightarrow+\infty} g\left(t-s_{n}\right)=\phi(t)
$$

uniformly on compact subsets of $\mathbb{R}$.

We denote by $A A_{c}(\mathbb{R} ; X)$, the space of all such functions.

It is well known that $A A_{c}(\mathbb{R} ; X)$ is closed subsets of $\left(B C(\mathbb{R}, X),|\cdot|_{\infty}\right)$.

In view of the above, the proof of the next lemma is straightforward.

Lemma 4.5. [7] $A A_{c}(\mathbb{R} ; X)$ equipped with the sup norm is a Banach space.

Definition 4.6. Let $X_{1}$ and $X_{2}$ be two Banach spaces. A continuous function $\phi: \mathbb{R} \times X_{1} \rightarrow X_{2}$ is called compact almost automorphic in $t \in \mathbb{R}$ if every real sequence $\left(s_{m}\right)$, there exists a subsequence $\left(s_{n}\right)$ such that

$$
g(t, x)=\lim _{n \rightarrow+\infty} \phi\left(t+s_{n}, x\right) \text { and } \lim _{n \rightarrow+\infty} g\left(t-s_{n}, x\right)=\phi(t, x) \text { in } X_{2}
$$

where the limits are uniform on compact subsets of $\mathbb{R}$ for each $x \in X_{1}$.

Denote by $A A_{c}\left(\mathbb{R} \times X_{1} ; X_{2}\right)$ the space of all such functions.

In the sequel, we recall some preliminary results concerning the $\alpha-(\mu, v)$-pseudo almost automorphic functions.

$\mathcal{E}\left(\mathbb{R} ; X_{\alpha}, \mu, v\right)$ stands for the space of functions

$$
\mathcal{E}\left(\mathbb{R} ; X_{\alpha}, \mu, v\right)=\left\{u \in B C\left(\mathbb{R} ; X_{\alpha}\right): \lim _{\tau \rightarrow+\infty} \frac{1}{v([-\tau, \tau])} \int_{-\tau}^{+\tau}|u(t)|_{\alpha} d \mu(t)=0\right\} .
$$

To study delayed differential equations for which the history belongs to $C\left([-r, 0] ; X_{\alpha}\right)$, we need to introduce the space

$$
\mathcal{E}\left(\mathbb{R} ; X_{\alpha}, \mu, \nu, r\right)=\left\{u \in B C\left(\mathbb{R} ; X_{\alpha}\right): \lim _{\tau \rightarrow+\infty} \frac{1}{v([-\tau, \tau])} \int_{-\tau}^{+\tau}\left(\sup _{\theta \in[t-r, t]}|u(\theta)| \alpha\right) d \mu(t)=0\right\} .
$$

In addition to above-mentioned spaces, we consider the following spaces

$$
\begin{gathered}
\mathcal{E}\left(\mathbb{R} \times X_{\alpha}, \mu, v\right)=\left\{u \in B C\left(\mathbb{R} \times X_{\alpha} ; X_{\alpha}\right): \lim _{\tau \rightarrow+\infty} \frac{1}{v([-\tau, \tau])} \int_{-\tau}^{+\tau}|u(t, x)|_{\alpha} d \mu(t)=0\right\}, \\
\mathcal{E}\left(\mathbb{R} \times X_{\alpha}, \mu, v, r\right)=\left\{u \in B C\left(\mathbb{R} \times X_{\alpha} ; X_{\alpha}\right): \lim _{\tau \rightarrow+\infty} \frac{1}{v([-\tau, \tau])} \int_{-\tau}^{+\tau}\left(\sup _{\theta \in[t-r, t]}|u(\theta, x)| \alpha\right) d \mu(t)=0\right\},
\end{gathered}
$$

where in both cases the limit (as $\tau \rightarrow+\infty$ ) is uniform in compact subset of $X_{\alpha}$.

In view of previous definitions, it is clear that the space $\mathcal{E}\left(\mathbb{R} ; X_{\alpha}, \mu, v, r\right)$ is continuously embedded in $\mathcal{E}\left(\mathbb{R} ; X_{\alpha}, \mu, v\right)$.

On the other hand, one can observe that a $\rho$-weighted pseudo almost automorphic functions is $\mu$-pseudo almost automorphic, where the measure $\mu$ is absolutely continuous with respect to the Lebesgue measure and its Radon-Nikodym derivative is $\rho$ :

$$
d \mu(t)=\rho(t) d t
$$

and $v$ is the usual Lebesgue measure on $\mathbb{R}$, i.e $v([-\tau, \tau])=2 \tau$ for all $\tau \geq 0$. 
Example 4.7. [3] Let $\rho$ be a nonnegative $\mathcal{B}$-measurable function. Denote by $\mu$ the positive measure defined by

$$
\mu(A)=\int_{A} \rho(t) d t, \text { for } A \in \mathcal{B},
$$

where dt denotes the Lebesgue measure on $\mathbb{R}$. The function $\rho$ which occurs in equation (3) is called the RadonNikodym derivative of $\mu$ with respect to the Lebesgue measure on $\mathbb{R}$.

Definition 4.8. A bounded continuous function $\phi: \mathbb{R} \rightarrow X$ is called $\alpha-(\mu, v)$-pseudo almost automorphic if $\phi=\phi_{1}+\phi_{2}$ where $\phi_{1} \in A A\left(\mathbb{R} ; X_{\alpha}\right)$ and $\phi_{2} \in \mathcal{E}\left(\mathbb{R} ; X_{\alpha}, \mu, \nu\right)$.

We denote by $P A A\left(\mathbb{R} ; X_{\alpha}, \mu, v\right)$, the space of all such functions.

Definition 4.9. A bounded continuous function $\phi: \mathbb{R} \times X_{\alpha} \rightarrow X_{\alpha}$ is called uniformly $\alpha-(\mu, v)$-pseudo almost automorphic if $\phi=\phi_{1}+\phi_{2}$, where $\phi_{1} \in A A\left(\mathbb{R} \times X_{\alpha} ; X_{\alpha}\right)$ and

$\phi_{2} \in \mathcal{E}\left(\mathbb{R} \times X_{\alpha} ; X_{\alpha}, \mu, v\right)$.

We denote by $P A A\left(\mathbb{R} \times X_{\alpha} ; X_{\alpha}, \mu, v\right)$, the space of all such functions.

We now introduce some new spaces used in the sequel.

Definition 4.10. A bounded continuous function $\phi: \mathbb{R} \rightarrow X$ is called compact $\alpha-(\mu, v)$-pseudo almost automorphic if $\phi=\phi_{1}+\phi_{2}$ where $\phi_{1} \in A A_{c}\left(\mathbb{R} ; X_{\alpha}\right)$ and $\phi_{2} \in \mathcal{E}\left(\mathbb{R} ; X_{\alpha}, \mu, v\right)$.

We denote by $P A A_{c}\left(\mathbb{R} ; X_{\alpha}, \mu, v\right)$, the space of all such functions.

Definition 4.11. A bounded continuous function $\phi: \mathbb{R} \times X_{\alpha} \rightarrow X_{\alpha}$ is called uniformly compact $\alpha-(\mu, v)$-pseudo almost automorphic if $\phi=\phi_{1}+\phi_{2}$, where $\phi_{1} \in A A_{c}\left(\mathbb{R} \times X_{\alpha} ; X_{\alpha}\right)$ and $\phi_{2} \in \mathcal{E}\left(\mathbb{R} \times X_{\alpha} ; X_{\alpha}, \mu, v\right)$.

We denote by $P A A_{c}\left(\mathbb{R} \times X_{\alpha} ; X_{\alpha}, \mu, v\right)$, the space of all such functions.

Definition 4.12. A bounded continuous function $\phi: \mathbb{R} \rightarrow X$ is called $\alpha-(\mu, v)$-pseudo almost automorphic of class $r$ (respectively compact $\alpha-(\mu, v)$-pseudo almost automorphic of class $r$ ) if $\phi=\phi_{1}+\phi_{2}$ where $\phi_{1} \in A A\left(\mathbb{R} ; X_{\alpha}\right)$ and $\phi_{2} \in \mathcal{E}\left(\mathbb{R} ; X_{\alpha}, \mu, \nu, r\right)$ (respectively if $\phi=\phi_{1}+\phi_{2}$ where $\phi_{1} \in A A_{c}\left(\mathbb{R} ; X_{\alpha}\right)$ and $\phi_{2} \in \mathcal{E}\left(\mathbb{R} ; X_{\alpha}, \mu, v, r\right)$.

We denote by $P A A\left(\mathbb{R} ; X_{\alpha}, \mu, v, r\right)$ (respectively $P A A_{c}\left(\mathbb{R} ; X_{\alpha}, \mu, v, r\right)$ ) the space of all such functions.

Definition 4.13. A bounded continuous function $\phi: \mathbb{R} \times X_{\alpha} \rightarrow X_{\alpha}$ is called uniformly pseudo almost automorphic of class $r$ (respectively uniformly pseudo compact almost automorphic of class $r$ ) if $\phi=\phi_{1}+\phi_{2}$, where $\phi_{1} \in A A\left(\mathbb{R} \times X_{\alpha} ; X_{\alpha}\right)$ and $\phi_{2} \in \mathcal{E}\left(\mathbb{R} \times X_{\alpha} ; X_{\alpha}, \mu, v, r\right)$ (respectively if $\phi=\phi_{1}+\phi_{2}$, where $\phi_{1} \in A A_{c}\left(\mathbb{R} \times X_{\alpha} ; X_{\alpha}\right)$ and $\phi_{2} \in \mathcal{E}\left(\mathbb{R} \times X_{\alpha} ; X_{\alpha}, \mu, v, r\right)$.

We denote by $P A A\left(\mathbb{R} \times X_{\alpha} ; X_{\alpha}, \mu, v, r\right)$ (respectively $\left.P A A_{c}\left(\mathbb{R} \times X_{\alpha} ; X_{\alpha}, \mu, v, r\right)\right)$ the space of all such functions.

\section{Properties of $(\mu, v)$-pseudo almost automorphic functions of class $r$}

From $\mu, v \in \mathcal{M}$, we formulate the following hypothese.

$\left(\mathbf{H}_{\mathbf{2}}\right)$ Let $\mu, v \in \mathcal{M}$ be such that $\limsup _{\tau \rightarrow+\infty} \frac{\mu([-\tau, \tau])}{v([-\tau, \tau])}=\delta<\infty$. 
We have the following result.

Lemma 5.1. Asumme $\left(\boldsymbol{H}_{2}\right)$ holds and let $f \in B C\left(\mathbb{R} ; X_{\alpha}\right)$. Then $f \in \mathcal{E}\left(\mathbb{R} ; X_{\alpha}, \mu, v\right)$ if and only if for any $\varepsilon>0$,

$$
\lim _{\tau \rightarrow+\infty} \frac{\mu\left(M_{\tau, \varepsilon}(f)\right)}{v([-\tau, \tau]}=0
$$

where

$$
M_{\tau, \varepsilon}(f)=\left\{t \in[-\tau, \tau]:|f(t)|_{\alpha} \geq \varepsilon\right\} .
$$

Proof. Suppose that $f \in \mathcal{E}\left(\mathbb{R} ; X_{\alpha}, \mu, v\right)$. Then

$$
\begin{aligned}
\frac{1}{v([-\tau, \tau])} \int_{-\tau}^{+\tau}|f(t)|_{\alpha} d \mu(t) & =\frac{1}{v([-\tau, \tau])} \int_{M_{\tau, \varepsilon}(f)}|f(t)|_{\alpha} d \mu(t)+\frac{1}{v([-\tau, \tau])} \int_{[-\tau, \tau] \backslash M_{\tau, \varepsilon}(f)}|f(t)|_{\alpha} d \mu(t) \\
& \geq \frac{1}{v([-\tau, \tau])} \int_{M_{\tau, \varepsilon}(f)}|f(t)|_{\alpha} d \mu(t) \\
& \geq \frac{\varepsilon \mu\left(M_{\tau, \varepsilon}(f)\right)}{v([-\tau, \tau])} .
\end{aligned}
$$

Consequently

$$
\lim _{\tau \rightarrow+\infty} \frac{\mu\left(M_{\tau, \varepsilon}(f)\right)}{v([-\tau, \tau]}=0 .
$$

Suppose that $f \in B C\left(\mathbb{R} ; X_{\alpha}\right)$ such that for any $\varepsilon>0$,

$$
\lim _{\tau \rightarrow+\infty} \frac{\mu\left(M_{\tau, \varepsilon}(f)\right)}{v([-\tau, \tau]}=0 .
$$

We can assume $|f(t)|_{\alpha} \leq N$ for all $t \in \mathbb{R}$. Using $\left(\mathbf{H}_{\mathbf{2}}\right)$, we have

$$
\begin{aligned}
\frac{1}{v([-\tau, \tau])} \int_{-\tau}^{+\tau}|f(t)|_{\alpha} d \mu(t) & =\frac{1}{v([-\tau, \tau])} \int_{M_{\tau, \varepsilon}(f)}|f(t)|_{\alpha} d \mu(t)+\frac{1}{v([-\tau, \tau])} \int_{[-\tau, \tau] \backslash M_{\tau, \varepsilon}(f)}|f(t)| \alpha d \mu(t) \\
& \leq \frac{N}{v([-\tau, \tau])} \int_{M_{\tau, \varepsilon}(f)} d \mu(t)+\frac{1}{v([-\tau, \tau])} \int_{[-\tau, \tau] \backslash M_{\tau, \varepsilon}(f)}|f(t)|_{\alpha} d \mu(t) \\
& \leq \frac{N}{v([-\tau, \tau])} \int_{M_{\tau, \varepsilon}(f)} d \mu(t)+\frac{\varepsilon}{v([-\tau, \tau])} \int_{[-\tau, \tau]} d \mu(t) \\
& \leq \frac{N}{v([-\tau, \tau])} \mu\left(M_{\tau, \varepsilon}(f)\right)+\frac{\varepsilon \mu([-\tau, \tau])}{v([-\tau, \tau])} .
\end{aligned}
$$

Which implies that

$$
\lim _{\tau \rightarrow+\infty} \frac{1}{v([-\tau, \tau])} \int_{-\tau}^{+\tau}|f(t)| \alpha d \mu(t) \leq \delta \varepsilon \text { for any } \varepsilon>0 .
$$

Therefore $f \in \mathcal{E}\left(\mathbb{R} ; X_{\alpha}, \mu, v\right)$

Lemma 5.2. Assume that $\left(\boldsymbol{H}_{2}\right)$ holds. The space $\mathcal{E}\left(\mathbb{R} ; X_{\alpha}, \mu, v, r\right)$ endowed with the uniform topology norm is a Banach space. 
Proof. We can see that $\mathcal{E}\left(\mathbb{R} ; X_{\alpha}, \mu, v, r\right)$ is a vector subspace of $B C\left(\mathbb{R} ; X_{\alpha}\right)$. To complete the proof, it is enough to prove that $\mathcal{E}\left(\mathbb{R} ; X_{\alpha}, \mu, v, r\right)$ is closed in $B C\left(\mathbb{R} ; X_{\alpha}\right)$. Let $\left(z_{n}\right)_{n}$ be a sequence in $\mathcal{E}\left(\mathbb{R} ; X_{\alpha}, \mu, v, r\right)$ such that $\lim _{n \rightarrow+\infty} z_{n}=z$ uniformly in $\mathbb{R}$. From $v(\mathbb{R})=+\infty$, it follows $v([-\tau, \tau])>0$ for $\tau$ sufficiently large. Let $\|z\|_{\infty, \alpha}=$ $\sup _{t \in \mathbb{R}}|z(t)|_{\alpha}$ and $n_{0} \in \mathbb{N}$ such that for all $n \geq n_{0},\left\|z_{n}-z\right\|_{\infty, \alpha}<\varepsilon$. Let $n \geq n_{0}$, then we have

$$
\begin{aligned}
\frac{1}{v([-\tau, \tau])} \int_{-\tau}^{+\tau}\left(\sup _{\theta \in[t-r, t]}|z(\theta)| \alpha\right) d \mu(t) \leq & \frac{1}{v([-\tau, \tau])} \int_{-\tau}^{+\tau}\left(\sup _{\theta \in[t-r, t]}\left|z_{n}(\theta)-z(\theta)\right| \alpha\right) d \mu(t) \\
& +\frac{1}{v([-\tau, \tau])} \int_{-\tau}^{+\tau}\left(\sup _{\theta \in[t-r, t]}\left|z_{n}(\theta)\right|_{\alpha}\right) d \mu(t) \\
\leq & \frac{1}{v([-\tau, \tau])} \int_{-\tau}^{+\tau}\left(\sup _{t \in \mathbb{R}}\left|z_{n}(t)-z(t)\right|_{\alpha}\right) d \mu(t) \\
& +\frac{1}{v([-\tau, \tau])} \int_{-\tau}^{+\tau}\left(\sup _{\theta \in[t-r, t]}\left|z_{n}(\theta)\right|_{\alpha}\right) d \mu(t) \\
\leq & \left\|z_{n}-z\right\|_{\infty, \alpha} \times \frac{\mu([-\tau, \tau])}{v([-\tau, \tau])}+\frac{1}{v([-\tau, \tau])} \int_{-\tau}^{+\tau}\left(\sup _{\theta \in[t-r, t]}\left|z_{n}(\theta)\right|_{\alpha}\right) d \mu(t) .
\end{aligned}
$$

We deduce that

$$
\limsup _{\tau \rightarrow+\infty} \frac{1}{v([-\tau, \tau])} \int_{-\tau}^{+\tau}\left(\sup _{\theta \in[t-r, t]}|z(\theta)| \alpha\right) d \mu(t) \leq \delta \varepsilon \text { for any } \varepsilon>0
$$

From the definition of $P A A\left(\mathbb{R} ; X_{\alpha}, \mu, v, r\right)$, we deduce the following result.

Proposition 5.3. Assume that $\left(\boldsymbol{H}_{2}\right)$ holds and let $\mu, v \in \mathcal{M}$. The space $P A A\left(\mathbb{R} ; X_{\alpha}, \mu, v, r\right)$ endowed with the uniform topology norm is a Banach space.

Next result is a characterization of $\alpha-(\mu, v)$-ergodic functions of class $r$.

Theorem 5.4. Assume that $\left(\mathrm{H}_{2}\right)$ holds and let $\mu, v \in \mathcal{M}$ and I be a bounded interval (eventually $I=$ ). Assume that $f \in B C\left(\mathbb{R}, X_{\alpha}\right)$. Then the following assertions are equivalent:

i) $f \in \mathcal{E}\left(\mathbb{R}, X_{\alpha}, \mu, v, r\right)$

ii) $\lim _{\tau \rightarrow+\infty} \frac{1}{v([-\tau, \tau] \backslash I)} \int_{[-\tau, \tau] \backslash I}\left(\sup _{\theta \in[t-r, t]}|f(\theta)| \alpha\right) d \mu(t)=0$.

iii) For any $\varepsilon>0, \lim _{\tau \rightarrow+\infty} \frac{\mu\left(\left\{t \in[-\tau, \tau] \backslash I: \sup _{\theta \in[t-r, t]}|f(\theta)| \alpha>\varepsilon\right\}\right)}{v([-\tau, \tau] \backslash I)}=0$.

Proof. $i) \Leftrightarrow$ ii) Denote by $A=v(I), B=\int_{I}\left(\sup _{\theta \in[t-r, t]}|f(\theta)| \alpha\right) d \mu(t)$. We have $A$ and $B \in \mathbb{R}$, since the interval $I$ is bounded and the function $f$ is bounded and continuous. For $\tau>0$ such that $I \subset[-\tau, \tau]$ and $v([-\tau, \tau] \backslash I)>0$, we have

$$
\begin{aligned}
& \frac{1}{v([-\tau, \tau] \backslash I)} \int_{[-\tau, \tau] \backslash I}\left(\sup _{\theta \in[t-r, t]}|f(\theta)| \alpha\right) d \mu(t)=\frac{1}{v([-\tau, \tau])-A}\left[\int_{[-\tau, \tau]}\left(\sup _{\theta \in[t-r, t]}|f(\theta)| \alpha\right) d \mu(t)-B\right] \\
= & \frac{v([-\tau, \tau])}{v([-\tau, \tau])-A}\left[\frac{1}{v([-r, r])} \int_{[-\tau, \tau]}\left(\sup _{\theta \in[t-r, t]}|f(\theta)| \alpha\right) d \mu(t)-\frac{B}{v([-\tau, \tau])}\right] .
\end{aligned}
$$


From above equalities and the fact that $v(\mathbb{R})=+\infty$, we deduce that $i i)$ is equivalent to

$$
\lim _{\tau \rightarrow+\infty} \frac{1}{v([-\tau, \tau])} \int_{-\tau}^{+\tau}\left(\sup _{\theta \in[t-r, t]}|f(\theta)| \alpha\right) d \mu(t)=0,
$$

that is $i)$.

iii) $\Rightarrow$ ii) Denote by $A_{\tau}^{\varepsilon}$ and $B_{\tau}^{\varepsilon}$ the following sets

$$
\left.A_{\tau}^{\varepsilon}=\left\{t \in[-\tau, \tau] \backslash I: \sup _{\theta \in[t-r, t]}|f(\theta)| \alpha>\varepsilon\right\} \text { and } B_{\tau}^{\varepsilon}=\{t \in[-\tau, \tau] \backslash I): \sup _{\theta \in[t-r, t]}|f(\theta)| \alpha \leq \varepsilon\right\} .
$$

Assume that iii) holds, that is

$$
\lim _{\tau \rightarrow+\infty} \frac{\mu\left(A_{\tau}^{\varepsilon}\right)}{v([-\tau, \tau] \backslash I)}=0
$$

From the equality

$$
\int_{[-\tau, \tau] \backslash I}\left(\sup _{\theta \in[t-r, t]}|f(\theta)| \alpha\right) d \mu(t)=\int_{A_{\tau}^{\varepsilon}}\left(\sup _{\theta \in[t-r, t]}|f(\theta)| \alpha\right) d \mu(t)+\int_{B_{\tau}^{\varepsilon}}\left(\sup _{\theta \in[t-r, t]}|f(\theta)|\right) d \mu(t),
$$

we deduce that for $\tau$ sufficiently large

$$
\frac{1}{v([-\tau, \tau] \backslash I)} \int_{[-\tau, \tau] \backslash I}\left(\sup _{\theta \in[t-r, t]}|f(\theta)|_{\alpha}\right) d \mu(t) \leq\|f\|_{\infty, \alpha} \frac{\mu\left(A_{\tau}^{\varepsilon}\right)}{v([-\tau, \tau] \backslash I)}+\varepsilon \frac{\mu\left(B_{\tau}^{\varepsilon}\right)}{v([-\tau, \tau] \backslash I)} .
$$

By using $\left(\mathbf{H}_{\mathbf{2}}\right)$, it follows that

$$
\lim _{\tau \rightarrow+\infty} \frac{1}{v([-\tau, \tau])} \int_{-\tau}^{+\tau}\left(\sup _{\theta \in[t-r, t]}|f(\theta)|_{\alpha}\right) d \mu(t) \leq \delta \varepsilon, \text { for any } \varepsilon>0,
$$

consequently (ii) holds.

ii) $\Rightarrow$ iii) Assume that ii) holds. From the following inequality

$$
\begin{aligned}
\int_{[-\tau, \tau] \backslash I}\left(\sup _{\theta \in[t-r, t]}|f(\theta)|_{\alpha}\right) d \mu(t) & \geq \int_{A_{\tau}^{\varepsilon}}\left(\sup _{\theta \in[t-r, t]}|f(\theta)| \alpha\right) d \mu(t) \\
\frac{1}{v([-\tau, \tau] \backslash I)} \int_{[-\tau, \tau] \backslash I}\left(\sup _{\theta \in[t-r, t]}|f(\theta)|_{\alpha}\right) d \mu(t) & \geq \varepsilon \frac{\mu\left(A_{\tau}^{\varepsilon}\right)}{v([-\tau, \tau] \backslash I)} \\
\frac{1}{\varepsilon v([-\tau, \tau] \backslash I)} \int_{[-\tau, \tau] \backslash I}\left(\sup _{\theta \in[t-r, t]}|f(\theta)|_{\alpha}\right) d \mu(t) & \geq \frac{\mu\left(A_{\tau}^{\varepsilon}\right)}{v([-\tau, \tau] \backslash I)},
\end{aligned}
$$

for $\tau$ sufficiently large, we obtain equation (4), that is iii).

From $\mu \in \mathcal{M}$, we formulate the following hypotheses.

$\left(\mathbf{H}_{3}\right)$ For all $a, b$ and $c \in \mathbb{R}$, such that $0 \leq a<b \leq c$, there exist $\delta_{0}$ and $\alpha_{0}>0$ such that

$$
|\delta| \geq \delta_{0} \Rightarrow \mu(a+\delta, b+\delta) \geq \alpha_{0} \mu(\delta, c+\delta) .
$$

$\left(\mathbf{H}_{\mathbf{4}}\right)$ For all $\tau \in \mathbb{R}$, there exist $\beta>0$ and a bounded interval $I$ such that

$$
\mu(\{a+\tau: a \in A\} \leq \beta \mu(A) \text { when } A \in \mathcal{B} \text { satisfies } A \cap I=.
$$

We have the following results due to [3] 
Lemma 5.5. [3] Hypothesis $\left(\boldsymbol{H}_{\mathbf{4}}\right)$ implies $\left(\boldsymbol{H}_{3}\right)$.

Proposition 5.6. $[3,4] \mu, v \in \mathcal{M}$ satisfy $\left(\boldsymbol{H}_{3}\right)$ and $f \in P A A(\mathbb{R} ; X, \mu, v)$ be such that

$$
f=g+h
$$

where $g \in A A(\mathbb{R}, X)$ and $h \in \mathcal{E}(\mathbb{R}, X, \mu, v)$. Then

$$
\{g(t), t \in \mathbb{R}\} \subset \overline{\{f(t), t \in \mathbb{R}\}} \text { (the closure of the range of } f \text { ). }
$$

Corollary 5.7. [4] Assume that $\left(\boldsymbol{H}_{3}\right)$ holds. Then the decomposition of a $(\mu, v)$-pseudo almost automorphic function in the form $f=g+\phi$ where $g \in A A(\mathbb{R} ; X)$ and $\phi \in \mathcal{E}(\mathbb{R} ; X, \mu, v)$, is unique.

We deduce following result.

Proposition 5.8. Let $\mu, v \in \mathcal{M}$. Assume $\left(\boldsymbol{H}_{3}\right)$ holds. Then the decomposition of a $\alpha-(\mu, v)$-pseudo-almost periodic function $\phi=\phi_{1}+\phi_{2}$, where $\phi_{1} \in A A\left(\mathbb{R} ; X_{\alpha}\right)$ and $\phi_{2} \in \mathcal{E}\left(\mathbb{R} ; X_{\alpha}, \mu, v, r\right)$, is unique.

Proof. In fact, since as a consequence of Corollary 5.7, the decomposition of a $(\mu, v)$-pseudo-almost periodic function $\phi=\phi_{1}+\phi_{2}$, where $\phi_{1} \in A A\left(\mathbb{R} ; X_{\alpha}\right)$ and $\phi_{2} \in \mathcal{E}\left(\mathbb{R} ; X_{\alpha}, \mu, v\right)$, is unique. Since $P A A\left(\mathbb{R} ; X_{\alpha}, \mu, v\right) \subset$ $P A A(\mathbb{R} ; X, \mu, v)$ and $P A A\left(\mathbb{R} ; X_{\alpha}, \mu, v, r\right) \subset P A A\left(\mathbb{R} ; X_{\alpha}, \mu, v\right)$, we get the desired result.

Definition 5.9. Let $\mu_{1}, \mu_{2} \in \mathcal{M}$. We say that $\mu_{1}$ is equivalent to $\mu_{2}$, denoting this as $\mu_{1} \sim \mu_{2}$ if there exist constants $\alpha$ and $\beta>0$ and a bounded interval I (eventually $I=$ ) such that

$$
\alpha \mu_{1}(A) \leq \mu_{2}(A) \leq \beta \mu_{1}(A) \text {, when } A \in \mathcal{B} \text { satisfies } A \cap I=\text {. }
$$

From [3] is a binary equivalence relation on $\mathcal{M}$. The equivalence class of a given measure $\mu \in \mathcal{N}$ will then be denoted by

$$
\operatorname{cl}(\mu)=\{\varpi \in \mathcal{M}: \mu \sim \varpi\} .
$$

Theorem 5.10. Let $\mu_{1}, \mu_{2}, v_{1}, v_{2} \in \mathcal{M}$. If $\mu_{1} \sim \mu_{2}$ and $v_{1} \sim v_{2}$, then $\operatorname{PAA}\left(\mathbb{R} ; X_{\alpha}, \mu_{1}, v_{1}, r\right)=$ $P A A\left(\mathbb{R} ; X_{\alpha}, \mu_{2}, v_{2}, r\right)$.

Proof. Since $\mu_{1} \sim \mu_{2}$ and $v_{1} \sim v_{2}$ there exist some constants $\alpha_{1}, \alpha_{2}, \beta_{1}, \beta_{2}>0$ and a bounded interval I (eventually $I=$ ) such that $\alpha_{1} \mu_{1}(A) \leq \mu_{2}(A) \leq \beta_{1} \mu_{1}(A)$ and $\alpha_{2} v_{1}(A) \leq v_{2}(A) \leq \beta_{2} v_{1}(A)$ for each $A \in \mathcal{B}$ satisfies $A \cap I=$ i.e

$$
\frac{1}{\beta_{2} v_{1}(A)} \leq \frac{1}{v_{2}(A)} \leq \frac{1}{\alpha_{2} v_{1}(A)} .
$$

Since $\mu_{1} \sim \mu_{2}$ and $\mathcal{B}$ is the Lebesgue $\sigma$-field, we obtain for $\tau$ sufficiently large that

$$
\begin{aligned}
\frac{\alpha_{1} \mu_{1}\left(\left\{t \in[-\tau, \tau] \backslash I: \sup _{\theta \in[t-r, t]}|f(\theta)| \alpha>\varepsilon\right\}\right)}{\beta_{2} v_{1}([-\tau, \tau] \backslash I)} & \leq \frac{\mu_{2}\left(\left\{t \in[-\tau, \tau] \backslash I: \sup _{\theta \in[t-r, t]}|f(\theta)|_{\alpha}>\varepsilon\right\}\right)}{v_{2}([-\tau, \tau] \backslash I)} \\
& \leq \frac{\beta_{1} \mu_{1}\left(\left\{t \in[-\tau, \tau] \backslash I: \sup _{\theta \in[t-r, t]}|f(\theta)| \alpha>\varepsilon\right\}\right)}{\alpha_{2} v_{1}([-\tau, \tau] \backslash I)} .
\end{aligned}
$$

By using Theorem 5.4 we deduce that $\mathcal{E}\left(\mathbb{R}, X_{\alpha}, \mu_{1}, v_{1}, r\right)=\mathcal{E}\left(\mathbb{R}, X_{\alpha}, \mu_{2}, v_{2}, r\right)$. From the definition of a $(\mu, v)$-pseudo almost automorphic function, we deduce that $P A A\left(\mathbb{R} ; X_{\alpha}, \mu_{1}, v_{1}, r\right)=P A A\left(\mathbb{R} ; X_{\alpha}, \mu_{2}, v_{2}, r\right)$ 
Let $\mu, v \in \mathcal{M}$. We denote by

$$
\operatorname{cl}(\mu, v)=\left\{\varpi_{1}, \varpi_{2} \in \mathcal{M}: \mu \sim \varpi_{2} \text { and } v \sim \varpi_{2}\right\}
$$

In what follows, we prove some preliminary results concerning the composition of $(\mu, v)$-pseudo almost automorphic functions of class $r$.

Theorem 5.11. Let $\mu, v \in \mathcal{M}, \phi \in P A A\left(\mathbb{R} \times X_{\alpha}, \mu, v, r\right)$ and $h \in P A A\left(\mathbb{R} ; X_{\alpha}, \mu, v, r\right)$. Assume that there exists a function $L_{\phi}: \mathbb{R} \rightarrow[0,+\infty[$ such that

$$
\left|\phi\left(t, x_{1}\right)-\phi\left(t, x_{2}\right)\right| \leq L_{\phi}(t)\left|x_{1}-x_{2}\right| \alpha \text { for } t \in \mathbb{R} \quad \text { and for } x_{1}, x_{2} \in X_{\alpha} .
$$

If

$$
\limsup _{\tau \rightarrow+\infty} \frac{1}{v([-\tau, \tau])} \int_{-\tau}^{\tau}\left(\sup _{\theta \in[t-r, t]} L_{\phi}(\theta)\right) d \mu(t)<\infty \text { and } \lim _{\tau \rightarrow+\infty} \frac{1}{v([-\tau, \tau])} \int_{-\tau}^{+\tau}\left(\sup _{\theta \in[t-r, t]} L_{\phi}(\theta)\right) \xi(t) d \mu(t)=0
$$

for each $\xi \in \mathcal{E}(\mathbb{R}, \mathbb{R}, \mu, v)$, then the function $t \rightarrow \phi(t, h(t))$ belongs to $P A A\left(\mathbb{R} ; X_{\alpha}, \mu, v, r\right)$.

Proof. Assume that $\phi=\phi_{1}+\phi_{2}, h=h_{1}+h_{2}$ where $\phi_{1} \in A A\left(\mathbb{R} \times X_{\alpha} ; X_{\alpha}\right), \phi_{2} \in \mathcal{E}\left(\mathbb{R} \times X_{\alpha}, \mu, v, r\right)$ and $h_{1} \in A A\left(\mathbb{R} ; X_{\alpha}\right), h_{2} \in \mathcal{E}\left(\mathbb{R} ; X_{\alpha}, \mu, v, r\right)$. Consider the following decomposition

$$
\phi(t, h(t))=\phi_{1}\left(t, h_{1}(t)\right)+\left[\phi(t, h(t))-\phi\left(t, h_{1}(t)\right)\right]+\phi_{2}\left(t, h_{1}(t)\right) \text {. }
$$

Since $\phi_{1} \in A A\left(\mathbb{R} \times X_{\alpha} ; X_{\alpha}\right)$, then $\phi_{1}(t, x)$ is uniformly continuous on any bounded subset uniformly for $t \in \mathbb{R}$. Define the function $g$ on $\mathbb{R}$ by $g(t)=\phi_{1}\left(t, h_{1}(t)\right)$, then $g \in A A\left(\mathbb{R} ; X_{\alpha}\right)$. In fact since $h_{1} \in A A\left(\mathbb{R} ; X_{\alpha}\right)$ then for a given sequence $\left(s_{m}\right)_{m \in \mathbb{N}}$ of real numbers, we can extract a subsequence $\left(s_{n}\right)_{n \in \mathbb{N}}$ and $v \in B C\left(\mathbb{R} ; X_{\alpha}\right)$ such that

$$
v(t)=\lim _{n \rightarrow+\infty} h_{1}\left(t+s_{n}\right)
$$

is well defined for each $t \in \mathbb{R}$, and

$$
\lim _{n \rightarrow+\infty} v\left(t-s_{n}\right)=h_{1}(t)
$$

for each $t \in \mathbb{R}$. Since $\phi_{1} \in A A\left(\mathbb{R} \times X_{\alpha} ; X_{\alpha}\right)$, then

$$
u(t, v(t))=\lim _{n \rightarrow+\infty} \phi_{1}\left(t+s_{n}, v(t)\right)=\lim _{n \rightarrow+\infty} \phi_{1}\left(t+s_{n}, h_{1}\left(t+s_{n}\right)\right)=g\left(t+s_{n}\right)
$$

is well defined for each $t \in \mathbb{R}$, and

$$
\lim _{n \rightarrow+\infty} u\left(t-s_{n}, v\left(t-s_{n}\right)\right)=\phi_{1}\left(t, \lim _{n \rightarrow+\infty} v\left(t-s_{n}\right)\right)=\phi_{1}\left(t, h_{1}(t)\right)=g(t)
$$

for each $t \in \mathbb{R}$. We conclude that $g \in A A\left(\mathbb{R} ; X_{\alpha}\right)$.

It remains to prove that both $\phi(., h())-.\phi\left(., h_{1}().\right)$ and $\phi_{2}\left(., h_{1}().\right)$ belong to $\varepsilon(\mathbb{R} ; X \alpha, \mu, v, r)$. Consequently, using inequality (5), it follows that

$$
\begin{aligned}
\frac{\mu\left(\left\{t \in[-\tau, \tau]: \sup _{\theta \in[t-r, t]}\left|\phi(\theta, h(\theta))-\phi\left(\theta, h_{1}(\theta)\right)\right|_{\alpha}>\varepsilon\right\}\right)}{v([-\tau, \tau])} & \leq \frac{\mu\left(\left\{t \in[-\tau, \tau]: \sup _{\theta \in[t-r, t]}\left(L_{\phi}(\theta)\left|h_{2}(\theta)\right| \alpha\right)>\varepsilon\right\}\right)}{v([-\tau, \tau])} \\
& \leq \frac{\mu\left(\left\{t \in[-\tau, \tau]:\left(\sup _{\theta \in[t-r, t]} L_{\phi}(\theta)\right)\left(\sup _{\theta \in[t-r, t]}\left|h_{2}(\theta)\right| \alpha\right)>\varepsilon\right\}\right)}{v([-\tau, \tau])} .
\end{aligned}
$$

Since $h_{2}$ is $(\mu, v)$-ergodic of class $r$, Theorem 5.4 and equation (6) yield that for the above-mentioned $\varepsilon$, we have

$$
\lim _{\tau \rightarrow+\infty} \frac{\mu\left(\left\{t \in[-\tau, \tau]:\left(\sup _{\theta \in[t-r, t]} L_{\phi}(\theta)\right)\left(\sup _{\theta \in[t-r, t]}\left|h_{2}(\theta)\right| \alpha\right)>\varepsilon\right\}\right)}{v([-\tau, \tau])}=0
$$


and then we obtain

$$
\lim _{\tau \rightarrow+\infty} \frac{\mu\left(\left\{t \in[-\tau, \tau]: \sup _{\theta \in[t-r, t]}\left|\phi(\theta, h(\theta))-\phi\left(\theta, h_{1}(\theta)\right)\right|_{\alpha}>\varepsilon\right\}\right)}{v([-\tau, \tau])}=0 .
$$

By Theorem 5.4, equation (7) shows that $t \mapsto \phi(t, h(t))-\phi\left(t, h_{1}(t)\right)$ is $(\mu, v)$-ergodic of class $r$.

Now to complete the proof, it is enough to prove that $t \mapsto \phi_{2}(t, h(t))$ is $(\mu, v)$-ergodic of class $r$. Since $\phi_{2}$ is uniformly continuous on the compact set $K=\overline{\left\{h_{1}(t): t \in \mathbb{R}\right\}}$ with respect to the second variable $x$, we deduce that for given $\varepsilon>0$, there exists $\delta>0$ such that, for all $t \in \mathbb{R}, \xi_{1}$ and $\xi_{2} \in K$, one has

$$
\left|\xi_{1}-\xi_{2}\right| \leq \delta \Rightarrow\left|\phi_{2}\left(t, \xi_{1}\right)-\phi_{2}\left(t, \xi_{2}\right)\right|_{\alpha} \leq \varepsilon
$$

Therefore, there exist $n(\varepsilon)$ and $\left\{z_{i}\right\}_{i=1}^{n(\varepsilon)} \subset K$, such that

$$
K \subset \bigcup_{i=1}^{n(\varepsilon)} B_{\delta}\left(z_{i}, \delta\right)
$$

and then

$$
\left\|\phi_{2}\left(t, h_{1}(t)\right)\right\| \leq \varepsilon+\sum_{1}^{n(\varepsilon)}\left\|\phi_{2}\left(t, z_{i}\right)\right\|
$$

Since

$$
\forall i \in\{1, \ldots, n(\varepsilon)\}, \quad \lim _{\tau \rightarrow+\infty} \frac{1}{v([-\tau, \tau])} \int_{-\tau}^{\tau}\left(\sup _{\theta \in[t-r, t]}\left|\phi_{2}\left(\theta, z_{i}\right)\right|_{\alpha}\right) d \mu(t)=0
$$

we deduce that

$$
\forall \varepsilon>0, \quad \limsup _{\tau \rightarrow+\infty} \frac{1}{v([-\tau, \tau])} \int_{-\tau}^{\tau}\left(\sup _{\theta \in[t-r, t]}\left|\phi_{2}\left(\theta, h_{1}(t)\right)\right| \alpha\right) d \mu(t) \leq \varepsilon \delta,
$$

that implies

$$
\lim _{\tau \rightarrow+\infty} \frac{1}{v([-\tau, \tau])} \int_{-\tau}^{\tau}\left(\sup _{\theta \in[t-r, t]}\left|\phi_{2}\left(\theta, h_{1}(\theta)\right)\right| \alpha\right) d \mu(t)=0 .
$$

Consequently $t \mapsto \phi_{2}(t, h(t))$ is $(\mu, v)$-ergodic of class $r$

For $\mu \in \mathcal{M}$ and $\delta \in \mathbb{R}$, we denote $\mu_{\delta}$ the positive measure on $(\mathbb{R}, \mathcal{B})$ defined by

$$
\mu_{\delta}(A)=\mu([a+\delta: a \in A])
$$

Lemma 5.12. [3] Let $\mu \in \mathcal{M}$ satisfy $\left(\boldsymbol{H}_{\mathbf{4}}\right)$. Then the measures $\mu$ and $\mu_{\delta}$ are equivalent for all $\delta \in \mathbb{R}$.

Lemma 5.13. [3] $\left(\boldsymbol{H}_{\mathbf{4}}\right)$ implies

$$
\text { for all } \sigma>0 \limsup _{\tau \rightarrow+\infty} \frac{\mu([-\tau-\sigma, \tau+\sigma])}{\mu([-\tau, \tau])}<+\infty .
$$

We have the following result.

Theorem 5.14. Assume that $\left(\boldsymbol{H}_{\mathbf{4}}\right)$ holds. Let $\mu, v \in \mathcal{M}$ and $\phi \in P A A_{c}\left(\mathbb{R} ; X_{\alpha}, \mu, v, r\right)$, then the function $t \rightarrow \phi_{t}$ belongs to $P A A_{c}\left(C_{\alpha}, \mu, v, r\right)$.

Proof. Assume that $u=g+h$ where $g \in A A\left(\mathbb{R} ; X_{\alpha}\right)$ and $h \in \mathcal{E}\left(\mathbb{R} ; X_{\alpha}, \mu, v, r\right)$. We can see that $u_{t}=g_{t}+h_{t}$. We want to show that $g_{t} \in A A\left(\mathbb{R} ; X_{\alpha}\right)$ and $h_{t} \in \mathcal{E}\left(\mathbb{R} ; X_{\alpha}, \mu, v, r\right)$.

Firstly for a given sequence $\left(s_{m}\right)_{m \in \mathbb{N}}$ of real numbers, fix a subsequence $\left(s_{n}\right)_{n \in \mathbb{N}}$ and $v \in B C\left(\mathbb{R} ; X_{\alpha}\right)$ such that 
$g\left(s+s_{n}\right) \rightarrow v(s)$ uniformly on compact subsets of $\mathbb{R}$. Let $K \subset \mathbb{R}$ be an arbitrary compact and $L>0$ such that $K \subset[-L, L]$. For $\varepsilon>0$, fix $N_{\varepsilon, L} \in \mathbb{N}$ such that $\left|g\left(s+s_{n}\right)-v(s)\right|_{\alpha} \leq \varepsilon$ for $s \in[-L, L]$ whenever $n \geq N_{\varepsilon, L}$. For $t \in K$ and $n \geq N_{\varepsilon, L}$, we have

$$
\begin{aligned}
\left|g_{t+s_{n}}-v_{t}\right|_{\alpha} & \leq \sup _{\theta \in[-L, L]}\left|g\left(\theta+s_{n}\right)-v(\theta)\right|_{\alpha} \\
& \leq \varepsilon .
\end{aligned}
$$

In view of above, $g_{t+s_{n}}$ converges to $v_{t}$ uniformly on $K$. Similary, one can prove that $v_{t-s_{n}}$ converges to $u_{t}$ uniformly on $K$. Thus, the function $s \rightarrow g_{s}$ belongs to $A A_{c}\left(\mathbb{R} ; X_{\alpha}\right)$.

Let us denote by

$$
M_{\delta}(\tau)=\frac{1}{v_{\delta}([-\tau, \tau])} \int_{-\tau}^{+\tau}\left(\sup _{\theta \in[t-r, t]}|h(\theta)| \alpha\right) d \mu_{\delta}(t),
$$

where $\mu_{\delta}$ and $v_{\delta}$ are the positive measures defined by equation (8). By using Lemma 5.12, it follows that $\mu_{\delta}$ and $\mu$ are equivalent and $v_{\delta}$ and $v$ are also equivalent. Then by using Theorem 5.10 we have $\mathcal{E}\left(\mathbb{R} ; X_{\alpha}, \mu_{\delta}, v_{\delta}, r\right)=$ $\mathcal{E}\left(\mathbb{R} ; X_{\alpha}, \mu, v, r\right)$, therefore $h \in \mathcal{E}\left(\mathbb{R} ; X, \mu_{\delta}, v_{\delta}, r\right)$, that is

$$
\lim _{\tau \rightarrow+\infty} M_{\delta}(\tau)=0, \text { for all } \delta \in \mathbb{R} .
$$

On the other hand, for $r>0$ we have

$$
\begin{aligned}
& \frac{1}{v([-\tau, \tau])} \int_{-\tau}^{+\tau}\left(\sup _{\theta \in[t-r, t]}\left[\sup _{\xi \in[-r, 0]}|h(\theta+\xi)| \alpha\right]\right) d \mu(t) \leq \frac{1}{v([-\tau, \tau])} \int_{-\tau}^{+\tau}\left(\sup _{\theta \in[t-2 r, t]}|h(\theta)|_{\alpha}\right) d \mu(t) \\
\leq & \frac{1}{v([-\tau, \tau])} \int_{-\tau}^{+\tau}\left(\sup _{\theta \in[t-2 r, t-r]}|h(\theta)|_{\alpha}+\sup _{\theta \in[t-r, t]}|h(\theta)|_{\alpha}\right) d \mu(t) \\
\leq & \frac{1}{v([-\tau, \tau])} \int_{-\tau-r}^{+\tau-r}\left(\sup _{\theta \in[t-r, t]}|h(\theta)|_{\alpha}\right) d \mu(t+r)+\frac{1}{v([-\tau, \tau])} \int_{-\tau}^{+\tau}\left(\sup _{\theta \in[t-r, t]}|h(\theta)|_{\alpha}\right) d \mu(t) \\
\leq & \frac{1}{v([-\tau, \tau])} \int_{-\tau-r}^{+\tau+r}\left(\sup _{\theta \in[t-r, t]}|h(\theta)|_{\alpha}\right) d \mu(t+r)+\frac{1}{v([-\tau, \tau])} \int_{-\tau}^{+\tau}\left(\sup _{\theta \in[t-r, t]}|h(\theta)|_{\alpha}\right) d \mu(t) \\
\leq & {\left[\frac{v([-\tau-r, \tau+r])}{v([-\tau, \tau])}\right] \times \frac{1}{v([-\tau-r, \tau+r]} \int_{-\tau-r}^{+\tau+r}\left(\sup _{\theta \in[t-r, t]}|h(\theta)|_{\alpha}\right) d \mu(t+r) } \\
& +\frac{1}{v([-\tau, \tau])} \int_{-\tau}^{+\tau}\left(\sup _{\theta \in[t-r, t]}|h(\theta)| \alpha\right) d \mu(t) .
\end{aligned}
$$

Consequently

$$
\begin{aligned}
\frac{1}{v([-\tau, \tau])} \int_{-\tau}^{+\tau}\left(\sup _{\theta \in[t-r, t]}\left[\sup _{\xi \in[-r, 0]}|h(\theta+\xi)|_{\alpha}\right]\right) d \mu(t) \leq & {\left[\frac{v([-\tau-r, \tau+r])}{v([-\tau, \tau])}\right] \times M_{r}(\tau+r) } \\
& +\frac{1}{v([-\tau, \tau])} \int_{-\tau}^{+\tau}\left(\sup _{\theta \in[t-r, t]}|h(\theta)| \alpha\right) d \mu(t),
\end{aligned}
$$

which shows using Lemma 5.12 and Lemma 5.13 that $\phi_{t}$ belongs to $P A A_{c}\left(C_{\alpha}, \mu, v, r\right)$. Thus, we obtain the desired result.

Lemma 5.15. [3] Let $\mu, v \in \mathcal{M}$ satisfy $\left(\boldsymbol{H}_{\mathbf{4}}\right)$. Then $P A A(\mathbb{R}, X, \mu, v)$ is invariant by translation, that is $f \in$ $P A A(\mathbb{R}, X, \mu, v)$ implies $f_{\gamma} \in P A A(\mathbb{R}, X, \mu, v)$ for all $\gamma \in \mathbb{R}$.

Corollary 5.16. Let $\mu, v \in \mathcal{M}$ satisfy $\left(\boldsymbol{H}_{\mathbf{4}}\right)$. Then $\operatorname{PAA}\left(\mathbb{R}, X_{\alpha}, \mu, v, r\right)$ is invariant by translation, that is $f \in$ $P A A\left(\mathbb{R}, X_{\alpha}, \mu, v, r\right)$ implies $f_{\gamma} \in P A A\left(\mathbb{R}, X_{\alpha}, \mu, v, r\right)$ for all $\gamma \in \mathbb{R}$. 
Proof. It suffices to prove that $\mathcal{E}(\mathbb{R}, X, \mu, v, r)$ is invariant by translation. Let $f \in \mathcal{E}(\mathbb{R}, X, \mu, \nu, \infty)$ and $F^{t}(\theta)=$ $\sup _{\theta \in[t-r, t]}|f(\theta)|$. Then $F^{t} \in \mathcal{E}(\mathbb{R}, \mathbb{R}, \mu, v)$, but since $\mathcal{E}(\mathbb{R}, \mathbb{R}, \mu, v)$ is invariant by translation, it follows that

$$
\lim _{\tau \rightarrow+\infty} \frac{1}{v([-\tau, \tau])} \int_{-\tau}^{\tau} F^{t}(\theta+\gamma) d \mu(t)=\lim _{\tau \rightarrow+\infty} \frac{1}{v([-\tau, \tau])} \int_{-\tau}^{\tau} \sup _{\theta \in[t-r, t]}|f(\theta+\gamma)| d \mu(t)=0,
$$

which implies that $f(.+\gamma) \in P A A(\mathbb{R}, X, \mu, v, r)$

\section{$6(\mu, v)$-pseudo almost automorphic solutions of class $r$}

In what follows, we will be looking at the existence of bounded integral solutions of class $r$ of equation (1).

Proposition 6.1. [1] Assume that $\left(\boldsymbol{H}_{\boldsymbol{O}}\right)$ and $\left(\boldsymbol{H}_{1}\right)$ hold and the semigroup $(\mathcal{U}(t))_{t \geq 0}$ is hyperbolic. If $f$ is bounded on $\mathbb{R}$, then there exists a unique bounded solution $u$ of equation (1) on $\mathbb{R}$, given by

$$
u_{t}=\lim _{\lambda \rightarrow+\infty} \int_{-\infty}^{t} u^{s}(t-s) \Pi^{s}\left(\widetilde{B}_{\lambda} X_{0} f(s)\right) d s+\lim _{\lambda \rightarrow+\infty} \int_{+\infty}^{t} u^{u}(t-s) \Pi^{u}\left(\widetilde{B}_{\lambda} X_{0} f(s)\right) d s \text { for } t \in \mathbb{R},
$$

where $\widetilde{B}_{\lambda}=\lambda\left(\lambda I-\mathcal{A}_{\mathcal{U}}\right)^{-1}$ for $\lambda>\widetilde{\omega}, \Pi^{s}$ and $\Pi^{u}$ are the projections of $C_{\alpha}$ onto the stable and unstable subspaces, respectively.

Proposition 6.2. Let $h \in A A_{c}(\mathbb{R} ; X)$ and $\Gamma$ be the mapping defined for $t \in \mathbb{R}$ by

$$
\Gamma h(t)=\left[\lim _{\lambda \rightarrow+\infty} \int_{-\infty}^{t} \mathcal{U}^{s}(t-s) \Pi^{s}\left(\widetilde{B}_{\lambda} X_{0} h(s)\right) d s+\lim _{\lambda \rightarrow+\infty} \int_{+\infty}^{t} \mathcal{U}^{u}(t-s) \Pi^{u}\left(\widetilde{B}_{\lambda} X_{0} h(s)\right) d s\right](0) .
$$

Then $\Gamma h \in A A_{c}\left(\mathbb{R}, X_{\alpha}\right)$.

Proof. We can see that $\Gamma h \in B C\left(\mathbb{R} ; X_{\alpha}\right)$. In fact

$$
\begin{aligned}
|\Gamma h(t)|_{\alpha} & \left.\leq \lim _{\lambda \rightarrow+\infty} \int_{-\infty}^{t}\left|\mathcal{U}^{s}(t-s) \Pi^{s}\left(\widetilde{B}_{\lambda} X_{0} h(s)\right)\right|_{\alpha} d s+\lim _{\lambda \rightarrow+\infty} \int_{t}^{+\infty}\left|\mathcal{U}^{u}(t-s) \Pi^{u}\left(\widetilde{B}_{\lambda} X_{0} h(s)\right)\right|_{\alpha} d s\right](0) \\
& \left.\leq \lim _{\lambda \rightarrow+\infty} \int_{-\infty}^{t}\left\|\mathcal{A}_{\mathcal{U}}^{\alpha} \mathcal{U}^{s}(t-s) \Pi^{s}\left(\widetilde{B}_{\lambda} X_{0} h(s)\right)\right\| d s+\lim _{\lambda \rightarrow+\infty} \int_{t}^{+\infty}\left\|\mathcal{A}_{\mathcal{U}}^{\alpha} \mathcal{u}^{u}(t-s) \Pi^{u}\left(\widetilde{B}_{\lambda} X_{0} h(s)\right)\right\| d s\right](0) \\
& \leq \bar{M} \widetilde{M} \int_{-\infty}^{t} \frac{e^{-\omega(t-s)}}{(t-s)^{\alpha}}\left|\Pi^{s}\right|\|h(s)\| d s+\bar{M} \widetilde{M} \int_{t}^{+\infty} \frac{e^{\omega(t-s)}}{(s-t)^{\alpha}}\left|\Pi^{u}\right|\|h(s)\| d s \\
& \leq \bar{M} \widetilde{M} \int_{-\infty}^{t} \frac{e^{-\omega(t-s)}}{(t-s)^{\alpha}}\left|\Pi^{s}\right|\|h(s)\| d s+\bar{M} \widetilde{M} \int_{t}^{+\infty} \frac{e^{\omega(t-s)}}{(s-t)^{\alpha}}\left|\Pi^{u}\right|\|h(s)\| d s \\
& \leq \bar{M} \widetilde{M} \int_{-\infty}^{t} \frac{e^{-\omega(t-s)}}{(t-s)^{\alpha}}\left|\Pi^{s}\right|\|h(s)\| d s+\bar{M} \widetilde{M} \int_{t}^{+\infty} \frac{e^{-\omega(s-t)}}{(s-t)^{\alpha}}\left|\Pi^{u}\right|\|h(s)\| d s \\
& \leq \frac{2 K\|h\|_{\infty}}{\omega^{1-\alpha}} \int_{0}^{+\infty} e^{-s} s^{-\alpha} d s=\frac{2 K\|h\|_{\infty} \Gamma(1-\alpha)}{\omega^{1-\alpha}}<\infty
\end{aligned}
$$

where $K=\max \left(\bar{M} \widetilde{M}\left|\Pi^{s}\right|, \bar{M} \widetilde{M}\left|\Pi^{u}\right|\right)$. 
For a given sequence $\left(s_{m}\right)_{m \in \mathbb{N}}$ of real numbers, fix a subsequence $\left(s_{n}\right)_{n \in \mathbb{N}}$ and $v \in B C\left(\mathbb{R} ; X_{\alpha}\right)$ such that $h\left(t+s_{n}\right)$ converges to $v(t)$ and $v\left(t-s_{n}\right)$ converges to $h(t)$ uniformly on compact subsets of $\mathbb{R}$. Then, if

$$
w\left(t+s_{n}\right)=\lim _{\lambda \rightarrow \infty} \int_{-\infty}^{t} u^{s}(t-s) \Pi^{s}\left(\widetilde{B}_{\lambda} X_{0} h\left(s+s_{n}\right)\right) d s+\lim _{\lambda \rightarrow \infty} \int_{+\infty}^{t} u^{u}(t-s) \Pi^{u}\left(\widetilde{B}_{\lambda} X_{0} h\left(s+s_{n}\right)\right) d s \text { for } t \in \mathbb{R}, n \in \mathbb{N},
$$

then by equation(9) and the Lebesgue Dominated Convergence Theorem, it follows that

$w\left(t+s_{n}\right)$ converges to

$$
z(t)=\lim _{\lambda \rightarrow+\infty} \int_{-\infty}^{t} u^{s}(t-s) \Pi^{s}\left(\widetilde{B}_{\lambda} X_{0} v(s)\right) d s+\lim _{\lambda \rightarrow+\infty} \int_{+\infty}^{t} u^{u}(t-s) \Pi^{u}\left(\widetilde{B}_{\lambda} X_{0} v(s)\right) d s
$$

It remains to prove that the convergence is uniform on all compact subset of $\mathbb{R}$. Let $K \subset \mathbb{R}$ be an arbitrary compact and let $\varepsilon>0$. Fix $L>0$ and $N_{\varepsilon} \in \mathbb{N}$ such that $K \subset\left[\frac{-L}{2}, \frac{L}{2}\right]$ with

$$
\begin{aligned}
\int_{\frac{L}{2}}^{+\infty} e^{-s} s^{-\alpha} & <\varepsilon \\
\left\|h\left(s+s_{n}\right)-v(s)\right\| & \leq \varepsilon \text { for } n \geq N_{\varepsilon} \text { and } s \in[-L, L] .
\end{aligned}
$$

Then, for each $t \in K$, ones has

$$
\begin{aligned}
& \left|w\left(t+s_{n}\right)-z(t)\right| \leq \bar{M} \widetilde{M}\left|\Pi^{s}\right| \int_{-\infty}^{t} \frac{e^{-\omega(t-s)}}{(t-s)^{\alpha}}\left\|h\left(s+s_{n}\right)-v(s)\right\| d s \\
& +\bar{M} \widetilde{M}\left|\Pi^{u}\right| \int_{t}^{+\infty} \frac{e^{-\omega(s-t)}}{(s-t)^{\alpha}}\left\|h\left(s+s_{n}\right)-v(s)\right\| d s \\
& \leq \bar{M} \widetilde{M}\left|\Pi^{s}\right| \int_{-\infty}^{-L} \frac{e^{-\omega(t-s)}}{(t-s)^{\alpha}}\left\|h\left(s+s_{n}\right)-v(s)\right\| d s \\
& +\bar{M} \widetilde{M}\left|\Pi^{s}\right| \int_{-L}^{t} \frac{e^{-\omega(t-s)}}{(t-s)^{\alpha}}\left\|h\left(s+s_{n}\right)-v(s)\right\| d s \\
& +\bar{M} \widetilde{M}\left|\Pi^{u}\right| \int_{t}^{+\infty} \frac{e^{-\omega(s-t)}}{(s-t)^{\alpha}}\left\|h\left(s+s_{n}\right)-v(s)\right\| d s \\
& \leq \frac{2 \bar{M} \widetilde{M}\left|\Pi^{s}\right|\|h\|_{\infty}}{\omega^{1-\alpha}} \int_{t+L}^{\infty} e^{-s} s^{-\alpha} d s+\frac{\bar{M} \tilde{M} \varepsilon\left(\left|\Pi^{s}+\right| \Pi^{u} \mid\right)}{\omega^{1-\alpha}} \int_{0}^{\infty} e^{-s} s^{-\alpha} d s \\
& \leq \frac{2 \bar{M} \widetilde{M}\left|\Pi^{s}\right|\|h\|_{\infty}}{\omega^{1-\alpha}} \int_{\frac{L}{2}}^{\infty} e^{-s} s^{-\alpha} d s+\frac{\bar{M} \widetilde{M} \varepsilon\left(\left|\Pi^{s}+\right| \Pi^{u} \mid\right)}{\omega^{1-\alpha}} \int_{0}^{\infty} e^{-\omega s} d s \\
& \leq \frac{2 \bar{M} \widetilde{M}}{\omega^{1-\alpha}}\left(2\left|\Pi^{s}\right|\|h\|_{\infty}+\left(\left|\Pi^{s}+\right| \Pi^{u} \mid\right) \int_{0}^{\infty} e^{-\omega s} d s\right) \varepsilon,
\end{aligned}
$$

which proves that the convergence is uniform on $K$, by the fact that the last estimate is independent of $t \in K$. Proceeding as previously, one can similarly prove that $z\left(t-s_{n}\right)$ converges to $w$ uniformly on compact subsets in $\mathbb{R}$. This completes the proof. 
Theorem 6.3. Let $\mu, v \in \mathcal{M}$ satisfy $\left(\boldsymbol{H}_{4}\right)$ and $g \in \mathcal{E}(\mathbb{R} ; X, \mu, v, r)$. Then $\Gamma g \in \mathcal{E}\left(\mathbb{R} ; X_{\alpha}, \mu, v, r\right)$.

Proof. In fact, for $\tau>0$ we get

$$
\begin{aligned}
\int_{-\tau}^{\tau}\left(\sup _{\theta \in[t-r, t]}|\Gamma g(\theta)|_{\alpha}\right) d \mu(t) \leq & \int_{-\tau}^{\tau}\left(\operatorname { s u p } _ { \theta \in [ t - r , t ] } \left[\lim _{\lambda \rightarrow+\infty} \int_{-\infty}^{\theta}\left|\mathcal{U}^{s}(\theta-s) \Pi^{s}\left(\widetilde{B}_{\lambda} X_{0} g(s)\right)\right|_{\alpha} d s\right.\right. \\
& \left.\left.+\lim _{\lambda \rightarrow+\infty} \int_{\theta}^{+\infty}\left|\mathcal{U}^{u}(\theta-s) \Pi^{u}\left(\widetilde{B}_{\lambda} X_{0} g(s)\right)\right| \alpha d s\right](0)\right) d \mu(t) \\
\leq & \int_{-\tau}^{\tau}\left(\operatorname { s u p } _ { \theta \in [ t - r , t ] } \left[\lim _{\lambda \rightarrow+\infty} \int_{-\infty}^{\theta}\left\|\mathcal{A}_{\mathcal{U}}^{\alpha} \mathcal{U}^{s}(\theta-s) \Pi^{s}\left(\widetilde{B}_{\lambda} X_{0} g(s)\right)\right\| d s\right.\right. \\
& \left.\left.+\lim _{\lambda \rightarrow+\infty} \int_{\theta}^{+\infty}\left\|\mathcal{A}_{\mathcal{U}}^{\alpha} \mathcal{U}^{u}(\theta-s) \Pi^{u}\left(\widetilde{B}_{\lambda} X_{0} g(s)\right)\right\| d s\right](0)\right) d \mu(t) \\
\leq & \bar{M} \widetilde{M} \int_{-\tau}^{\tau}\left(\sup _{\theta \in[t-r, t]} \int_{-\infty}^{\theta} \frac{e^{-\omega(\theta-s)}}{(\theta-s)^{\alpha}}\left|\Pi^{s}\right||g(s)| d s\right) d \mu(t) \\
& +\bar{M} \widetilde{M} \int_{-\tau}^{\tau}\left(\sup _{\theta \in[t-r, t]} \int_{\theta}^{+\infty} \frac{e^{\omega(\theta-s)}}{(s-\theta)^{\alpha}}\left|\Pi^{u}\right||g(s)| d s\right) d \mu(t) \\
\leq & K\left[\int_{-\tau}^{\tau}\left(\sup _{\theta \in[t-r, t]}^{\theta} \int_{-\infty}^{\theta} \frac{e^{-\omega(\theta-s)}}{(\theta-s)^{\alpha}}|g(s)| d s\right) d \mu(t)+\int_{-\tau}^{\tau}\left(\sup _{\theta \in[t-r, t]} \int_{\theta}^{+\infty} \frac{e^{\omega(\theta-s)}}{(s-\theta)^{\alpha}}|g(s)| d s\right) d \mu(t)\right],
\end{aligned}
$$

where $K=\max \left(\bar{M} \widetilde{M}\left|\Pi^{s}\right|, \bar{M} \widetilde{M}\left|\Pi^{u}\right|\right)$. On the one hand using Fubini's theorem, we have

$$
\begin{aligned}
\int_{-\tau}^{\tau}\left(\sup _{\theta \in[t-r, t]} \int_{-\infty}^{\theta} \frac{e^{-\omega(\theta-s)}}{(\theta-s)^{\alpha}}|g(s)| d s\right) d \mu(t) & \leq \int_{-\tau}^{\tau}\left(\sup _{\theta \in[t-r, t]} \int_{0}^{+\infty} \frac{e^{-\omega s}}{s^{\alpha}}|g(\theta-s)| d s\right) d \mu(t) \\
& \leq \int_{0}^{+\infty} \frac{e^{-\omega s}}{s^{\alpha}}\left(\sup _{\theta \in[t-r, t]} \int_{-\tau}^{\tau}|g(\theta-s)| d \mu(t)\right) d s .
\end{aligned}
$$

By the Lebesgue dominated convergence theorem and by using Corollary 5.16, it follows that

$$
\lim _{\tau \rightarrow+\infty} \int_{0}^{+\infty} \frac{e^{-\omega s}}{s^{\alpha}} \frac{1}{v([-\tau, \tau])}\left(\sup _{\theta \in[t-r, t]} \int_{-\tau}^{\tau}|g(\theta-s)| d \mu(t)\right) d s=0 .
$$

On the other hand by Fubini's theorem, we also have

$$
\begin{aligned}
\int_{-\tau}^{\tau}\left(\sup _{\theta \in[t-r, t]} \int_{\theta}^{+\infty} \frac{e^{\omega(\theta-s)}}{(s-\theta)^{\alpha}}|g(s)| d s\right) d \mu(t) & \leq \int_{-\tau}^{\tau}\left(\sup _{\theta \in[t-r, t]} \int_{0}^{+\infty} \frac{e^{-\omega s}}{s^{\alpha}}|g(s+\theta)| d s\right) d \mu(t) \\
& \leq \int_{0}^{+\infty} \frac{e^{-\omega s}}{s^{\alpha}}\left(\sup _{\theta \in[t-r, t]} \int_{-\tau}^{\tau}|g(s+\theta)| d \mu(t)\right) d s .
\end{aligned}
$$

Resoning like above, it follows that

$$
\lim _{\tau \rightarrow+\infty} \int_{0}^{+\infty} \frac{e^{-\omega s}}{s^{\alpha}}\left(\sup _{\theta \in[t-r, t]} \int_{-\tau}^{\tau}|g(s+\theta)| d \mu(t)\right) d s=0 .
$$

Consequently

$$
\lim _{\tau \rightarrow+\infty} \frac{1}{v([-\tau, \tau])} \int_{-\tau}^{\tau}\left(\sup _{\theta \in[t-r, t]}|(\Gamma g)(\theta)| \alpha\right) d \mu(t)=0
$$


Thus, we obtain the desired result.

For the existence of $(\mu, v)$-pseudo almost automorphic solution of class $r$, we make the following assumption.

$\left(\mathbf{H}_{5}\right) f: \mathbb{R} \rightarrow X$ is compact $c l(\mu, v)$-pseudo almost automorphic of class $r$.

Proposition 6.4. Assume $\left(\boldsymbol{H}_{\mathbf{O}}\right),\left(\boldsymbol{H}_{1}\right),\left(\boldsymbol{H}_{3}\right)$ and $\left(\boldsymbol{H}_{5}\right)$ hold. Then equation (1) has a unique compact $\alpha-c l(\mu, v)$ pseudo almost automorphic solution of class $r$.

Proof. Since $f$ is a $(\mu, v)$-pseudo almost automorphic function, $f$ has a decomposition $f=f_{1}+f_{2}$ where $f_{1} \in A A_{c}(\mathbb{R} ; X)$ and $f_{2} \in \mathcal{E}(\mathbb{R} ; X, \mu, v, r)$. Using Proposition 6.1, Proposition 6.2 and Theorem 6.3, we get the desired result.

Our next objective is to show the existence of $(\mu, v)$-pseudo almost automorphic solutions of class $r$ for the following problem

$$
u^{\prime}(t)=-A u(t)+L\left(u_{t}\right)+f\left(t, u_{t}\right) \text { for } t \in \mathbb{R}
$$

where $f: \mathbb{R} \times C_{\alpha} \rightarrow X$ is continuous.

For the sequel, we make the following assumptions.

(Ho $)$ The instable space $U \equiv\{0\}$.

$\left(\mathbf{H}_{7}\right)$ Let $\mu, v \in \mathcal{M}$ and $f: \mathbb{R} \times C_{\alpha} \rightarrow X \operatorname{cl}(\mu, v)$-pseudo almost automorphic of class $r$ such that there exists a positive constant $L_{f}$ such that

$$
\left\|f\left(t, \varphi_{1}\right)-f\left(t, \varphi_{2}\right)\right\| \leq L_{f}\left\|\varphi_{1}-\varphi_{2}\right\|_{C_{\alpha}} \text { for all } t \in \mathbb{R} \text { and } \varphi_{1}, \varphi_{2} \in C_{\alpha}
$$

and $L_{f}$ satisfies (6).

Theorem 6.5. Assume $\left(\boldsymbol{H}_{0}\right),\left(\boldsymbol{H}_{1}\right),\left(\boldsymbol{H}_{2}\right),\left(\boldsymbol{H}_{3}\right),\left(\boldsymbol{H}_{6}\right)$ and $\left(\boldsymbol{H}_{7}\right)$ hold. If

$$
\frac{\bar{M} \widetilde{M}\left|\Pi^{s}\right| L_{f} \Gamma(1-\alpha)}{\omega^{1-\alpha}}<1,
$$

then equation (10) has a unique compact $\alpha-c l(\mu, v)$-pseudo almost automorphic solution of class $r$.

Proof. Let $x$ be a function in $P A A_{c}(\mathbb{R} ; X, \mu, v, r)$, from Theorem 5.14 the function $t \rightarrow x_{t}$ belongs to $P A A_{c}\left(C_{\alpha}, \mu, r\right)$. Hence Theorem 5.11 implies that the function $g():.=f(., x$.$) is in P A A(\mathbb{R} ; X, \mu, r)$. Since the unstable space $U \equiv\{0\}$, then $\Pi^{u} \equiv 0$. Consider the mapping

$$
\mathcal{H}: P A A_{c}\left(\mathbb{R} ; X_{\alpha}, \mu, v, r\right) \rightarrow P A A_{c}\left(\mathbb{R} ; X_{\alpha}, \mu, v, r\right)
$$

defined for $t \in \mathbb{R}$ by

$$
(\mathcal{H} x)(t)=\left[\lim _{\lambda \rightarrow+\infty} \int_{-\infty}^{t} U^{s}(t-s) \Pi^{s}\left(\widetilde{B}_{\lambda} X_{0} f\left(s, x_{s}\right)\right) d s\right](0) .
$$

From Proposition 6.1, Proposition 6.2 and taking into account Theorem 6.3, it suffices now to show that the operator $\mathcal{H}$ has a unique fixed point in $P A A\left(\mathbb{R} ; X_{\alpha}, \mu, r\right)$. 
Let $x_{1}, x_{2} \in P A A_{c}\left(\mathbb{R} ; X_{\alpha}, r\right)$. Then we have

$$
\begin{aligned}
\left|\mathcal{H} x_{1}(t)-\mathcal{H} x_{2}(t)\right|_{\alpha} & \leq\left|\lim _{\lambda \rightarrow+\infty} \int_{-\infty}^{t} \mathcal{U}^{s}(t-s) \Pi^{s}\left(\widetilde{B}_{\lambda} X_{0}\left(f\left(s, x_{1 s}\right)-f\left(s, x_{2 s}\right)\right)\right) d s\right|_{\alpha} \\
& \leq \bar{M} \widetilde{M}\left|\Pi^{s}\right| L_{f} \int_{-\infty}^{t} \frac{e^{-\omega(t-s)}}{(t-s)^{\alpha}}\left|x_{1 s}-x_{2 s}\right| d s \\
& \leq \bar{M} \widetilde{M}\left|\Pi^{s}\right|\left|x_{1}-x_{2}\right| L_{f} \int_{-\infty}^{t} \frac{e^{-\omega(t-s)}}{(t-s)^{\alpha}} d s \\
& \leq \frac{\bar{M} \widetilde{M}\left|\Pi^{s}\right| L_{f}}{\omega^{1-\alpha}}\left(\int_{0}^{+\infty} e^{-s} s^{-\alpha} d s\right)\left|x_{1}-x_{2}\right| \\
& \leq \frac{\bar{M} \widetilde{M}\left|\Pi^{s}\right| L_{f} \Gamma(1-\alpha)}{\omega^{1-\alpha}}\left|x_{1}-x_{2}\right| .
\end{aligned}
$$

This means that $\mathcal{H}$ is a strict contraction. Thus by Banach's fixed point theorem, $\mathcal{H}$ has a unique fixed point $u$ in $P A A_{c}\left(\mathbb{R} ; X_{\alpha}, \mu, v, r\right)$. We conclude that equation (10), has one and only one $c l(\mu, v)$-pseudo almost automorphic solution of class $r$

\section{Application}

For illustration, we propose to study the existence of solutions for the following model

$$
\left\{\begin{array}{l}
\frac{\partial z(t, x)}{\partial t}=-\frac{\partial^{2} z(t, x)}{\partial x^{2}}+\int_{-r}^{0} G(\theta) z(t+\theta, x) d \theta+\int_{0}^{t} \sin \left(\frac{1}{2+\cos s+\cos \sqrt{2} s}\right) d s \\
\quad+\arctan (t)+h\left(t, \frac{\partial}{\partial x} z(t+\theta, x)\right) \text { for } t \in \mathbb{R} \text { and } x \in[0, \pi] \\
z(t, 0)=z(t, \pi)=0 \text { for } t \in \mathbb{R},
\end{array}\right.
$$

where $G:[-r, 0] \rightarrow \mathbb{R}$ is a continuous function and $h: \mathbb{R} \times \mathbb{R} \rightarrow \mathbb{R}$ is Lipschitz continuous with respect to the second argument. To rewrite equation (11) in the abstract form, we introduce the space $X=L^{2}([0, \pi] ; \mathbb{R})$ vanishing at 0 and $\pi$, equipped with the $L^{2}$ norm that is to say for all $x \in X$,

$$
\|x\|_{L^{2}}=\left(\int_{0}^{\pi}|x(s)|^{2} d s\right)^{\frac{1}{2}} .
$$

Let $A: X \rightarrow X$ be defined by

$$
\left\{\begin{array}{l}
D(A)=H^{2}(0, \pi) \cap H_{0}^{1}(0, \pi) \\
A y=y^{\prime \prime} .
\end{array}\right.
$$

Then the spectrum $\sigma(A)$ of A equals to the point spectrum $\sigma_{p}(A)$ and is given by

$$
\sigma(A)=\sigma_{p}(A)=\left\{-n^{2}: n \geq 1\right\}
$$

and the associated eigenfunctions $\left(e_{n}\right)_{n \geq 1}$ are given by

$$
e_{n}(s)=\sqrt{\frac{2}{\pi}} \sin (n s), s \in[0, \pi] .
$$


Then the operator is computed by

$$
A y=\sum_{n=1}^{+\infty} n^{2}\left(y, e_{n}\right) e_{n}, y \in D(A)
$$

For each $y \in D\left(A^{\frac{1}{2}}\right)=\left\{y \in X: \sum_{n=1}^{+\infty} n\left(y, e_{n}\right) e_{n} \in X\right\}$, the operator $A^{\frac{1}{2}}$ is given by

$$
A^{\frac{1}{2}} y=\sum_{n=1}^{+\infty} n\left(y, e_{n}\right) e_{n}, y \in D(A)
$$

Lemma 7.1. [9] If $y \in D\left(A^{\frac{1}{2}}\right)$, then $y$ is absolutely continuous, $y^{\prime} \in X$ and $\left|y^{\prime}\right|=\left|A^{\frac{1}{2}} y\right|$.

It is well known that $-A$ is the generator of a compact analytic semigroup semigroup $(T(t))_{t \geq 0}$ on $X$ which is given by

$$
T(t) x=\sum_{n=1}^{+\infty} e^{-n^{2} t}\left(x, e_{n}\right) e_{n}, x \in X
$$

Then $\left(\mathbf{H}_{\mathbf{0}}\right)$ and $\left(\mathbf{H}_{\mathbf{1}}\right)$ are satified. Here we choose $\alpha=\frac{1}{2}$.

We define $f: \mathbb{R} \times C_{\frac{1}{2}} \rightarrow X$ and $L: C_{\frac{1}{2}} \rightarrow X$ as follows

$$
\begin{aligned}
f(t, \varphi)(x)= & \int_{0}^{t} \sin \left(\frac{1}{2+\cos s+\cos \sqrt{2} s}\right) d s+\arctan (t)+h\left(t, \frac{\partial}{\partial x} \varphi(\theta, x)\right) \text { for } x \in[0, \pi] \text { and } t \in \mathbb{R}, \\
L(\varphi)(x)= & \left.\int_{-r}^{0} G(\theta) \varphi(\theta, x)\right) d \theta \text { for }-r \leq \theta \leq 0 \text { and } x \in[0, \pi] .
\end{aligned}
$$

Let us pose $v(t)=z(t, x)$. Then equation (11) takes the following abstract form

$$
v^{\prime}(t)=-A v(t)+L\left(v_{t}\right)+f\left(t, v_{t}\right) \text { for } t \in \mathbb{R} .
$$

Consider the measures $\mu$ and $\nu$ where its Radon-Nikodym derivative are respectively $\rho_{1}, \rho_{2}: \mathbb{R} \rightarrow \mathbb{R}$ defined by

$$
\rho_{1}(t)=\left\{\begin{array}{l}
1 \text { for } t>0 \\
e^{t} \text { for } t \leq 0
\end{array}\right.
$$

and

$$
\rho_{2}(t)=|t| \text { for } t \in \mathbb{R}
$$

i.e $d \mu(t)=\rho_{1}(t) d t$ and $d v(t)=\rho_{2}(t) d t$ where $d t$ denotes the Lebesgue measure on $\mathbb{R}$ and

$$
\mu(A)=\int_{A} \rho_{1}(t) d t \text { for } v(A)=\int_{A} \rho_{2}(t) d t \text { for } A \in \mathcal{B} .
$$

From [?] $\mu, v \in \mathcal{M}, \mu, v$ satisfy Hypothesis $\left(\mathbf{H}_{\mathbf{4}}\right)$.

We have

$$
\limsup _{\tau \rightarrow+\infty} \frac{\mu([-\tau, \tau])}{v([-\tau, \tau])}=\limsup _{\tau \rightarrow+\infty} \frac{\int_{-\tau}^{0} e^{t} d t+\int_{0}^{\tau} d t}{2 \int_{0}^{\tau} t d t}=\limsup _{\tau \rightarrow+\infty} \frac{1-e^{-\tau}+\tau}{\tau^{2}}=0<\infty,
$$


which implies that $\left(\mathbf{H}_{\mathbf{2}}\right)$ is satisfied.

Since $A^{\frac{1}{2}}\left(\int_{0}^{t} \sin \left(\frac{1}{2+\cos s+\cos \sqrt{2} s}\right) d s\right)=\sin \left(\frac{1}{2+\cos t+\cos \sqrt{2} t}\right)$ and $t \rightarrow \sin \left(\frac{1}{2+\cos t+\cos \sqrt{2} t}\right)$ belongs to $A A_{c}(\mathbb{R}, X)$, it follows that $t \rightarrow \int_{0}^{t} \sin \left(\frac{1}{2+\cos s+\cos \sqrt{2} s}\right) d s$ belongs to $A A_{c}\left(\mathbb{R}, X_{\frac{1}{2}}\right)$. On the other hand, we have the following:

$$
\begin{aligned}
\frac{1}{v([-\tau, \tau])} \int_{-\tau}^{+\tau} \sup _{\theta \in[t-r, t]}|\arctan (\theta)|_{\frac{1}{2}} d t & =\frac{1}{v([-\tau, \tau])} \int_{-\tau}^{+\tau} \sup _{\theta \in[t-r, t]}\left|A^{\frac{1}{2}} \arctan (\theta)\right| d t \\
& =\frac{1}{v([-\tau, \tau])} \int_{-\tau}^{+\tau} \sup _{\theta \in[t-r, t]}\left(\frac{1}{1+\theta^{2}}\right) d t \\
& \leq \frac{\mu([-\tau, \tau])}{v([-\tau, \tau])} \rightarrow 0 \text { as } \tau \rightarrow+\infty .
\end{aligned}
$$

It follows that $t \mapsto \arctan t$ is $(\mu, v)$-ergodic of class $r$, consequently, $f$ is uniformly compact $(\mu, v)$-pseudo almost automorphic of class $r$. Moreover, $L$ is a bounded linear operator from $C_{\frac{1}{2}}$ to $X$.

Let $k$ be the lipschiz constant of $h$, then for every $\varphi_{1}, \varphi_{2} \in C_{\frac{1}{2}}$ and $t \geq 0$, we have

$$
\begin{aligned}
\left\|f\left(t, \varphi_{1}\right)(x)-f\left(t, \varphi_{2}\right)(x)\right\| & =\left(\int_{0}^{\pi}\left[h\left(\theta, \frac{\partial}{\partial x} \varphi_{1}(\theta, x)\right)-h\left(t, \frac{\partial}{\partial x} \varphi_{1}(t, x)\right)\right]^{2} d x\right)^{\frac{1}{2}} \\
& \leq L_{h}\left[\int_{0}^{\pi}\left(\frac{\partial}{\partial x} \varphi_{1}(\theta, x)-\frac{\partial}{\partial x} \varphi_{2}(\theta, x)\right)^{2} d x\right]^{\frac{1}{2}} \\
& \leq L_{h} \sup _{-r \leq \theta \leq 0}\left[\int_{0}^{\pi}\left(\frac{\partial}{\partial x} \varphi_{1}(\theta, x)-\frac{\partial}{\partial x} \varphi_{2}(\theta, x)\right)^{2} d x\right]^{\frac{1}{2}} \\
& \leq L_{h}\left\|\varphi_{1}-\varphi_{2}\right\|_{c_{\alpha}}
\end{aligned}
$$

Consequently, we conclude that $f$ is Lipschitz continuous and $c l(\mu, v)$-pseudo almost automorphic of class $r$.

Lemma 7.2. [6] If $\int_{-r}^{0}|G(\theta)| d \theta<1$, then the semigroup $(U(t))_{t \geq 0}$ is hyperbolic.

For example, let us pose $G(\theta)=\frac{\theta^{2}-1}{\left(\theta^{2}+1\right)^{2}}$ for $\theta \in[-r, 0]$. Then we can see that

$$
\int_{-r}^{0}|G(\theta)| d \theta=\int_{-r}^{0}\left|\frac{\theta^{2}-1}{\left(\theta^{2}+1\right)^{2}}\right| d \theta=\left[\frac{\theta}{\theta^{2}+1}\right]_{-r}^{0}=\frac{r}{r^{2}+1}<1 \text { if } r<1
$$

and

$$
\int_{-r}^{0}|G(\theta)| d \theta=\int_{-r}^{0}\left|\frac{\theta^{2}-1}{\left(\theta^{2}+1\right)^{2}}\right| d \theta=\int_{-r}^{-1} \frac{\theta^{2}-1}{\left(\theta^{2}+1\right)^{2}} d \theta+\int_{-1}^{0} \frac{-\theta^{2}+1}{\left(\theta^{2}+1\right)^{2}} d \theta=1-\frac{r}{r^{2}+1}<1 \text { if } r \geq 1,
$$

By Proposition 6.5 we deduce the following result.

Theorem 7.3. Under the above assumptions, if Lip ( $h$ ) is small enough, then equation (12) has a unique compact $\alpha-\operatorname{cl}(\mu, v)$-pseudo almost automorphic solution $v$ of class $r$. 


\section{References}

[1] M. Adimy, A. Elazzouzi and K. Ezzinbi, Reduction principle and dynamic behavoirs for a class of partial functional differential equations , Nonlinear Analysis, TMA, 71, (2009), 1709-1727.

[2] M. Adimy, K. Ezzinbi, M. Laklach, Spectral decomposition for partial neutral functional differential equations, Canadian Applied Mathematics Quarterly, (1), (2001), 1-34.

[3] J. Blot, P. Cieutat, K. Ezzinbi Measure theory and pseudo almost automorphic functions: New developments and applications, Nonlinear Analysis (2012), 2426-2447

[4] T. Diagana, K. Ezzinbi and M. Miraoui, Pseudo-almost periodic and pseudo-almost automorphic solutions to some evolution equations involving theoretical measure theory, CUBO A Mathematical Journal,16, (02), (2014), 01-31.

[5] K. Ezzinbi, H. Toure, I. Zabsonre PPseudo almost automorphic solutions of class $r$ for some partial functional differential equations, Afrika Matematika, (2014), 25-41.

[6] K. Ezzinbi, S. Fatajou, G.M. N'Guérékata, $C^{n}$-almost automorphic solutions for partial neutral functional differential equations, Applicable Analysis, 86:9, (2007), 1127-1146.

[7] G.M. N'Guérékata, Almost automorphic and almost periodic functions, Kluwer Academic Publishers, New York, (2001).

[8] A. Pazy, Semigroups of Linear Operators and Application to Partial Differental Equation, Applied Mathematical Sciences, Springer-Verlag, New York, Vol. 44, (1983).

[9] C.C. Travis and G.F. Webb, Existence, stability, and compactness in the $\alpha$-norm for partial functional differential equations , Transaction of the American Mathematical Society, 240, 129-143, (1978). 